\title{
Periodic Grating Structures on Metal Self-organized by Double-pulse Irradiation
}

\author{
Masaki HASHIDA ${ }^{* 1,{ }^{* 2}}$, Laura GEMINI ${ }^{* 1,{ }^{*},{ }^{*},{ }^{*} 4}$, Takaya NISHII ${ }^{* 1,{ }^{* 2}}$, Yasuhiro MIYASAKA ${ }^{* 1,{ }^{* 2}}$, Hitoshi SAKAGAMI ${ }^{* 5}$, \\ Masahiro SHIMIZU, Shunsuke INOUE ${ }^{* 1,{ }^{*}}$, Jiri LIMPOUCH ${ }^{* 3}$, Tomas Mocek ${ }^{* 4}$, and Shuji SAKABE ${ }^{* 1,{ }^{* 2}}$ \\ ${ }^{*}$ Advanced Research Center for Beam Science, Institute for Chemical Research, Kyoto University \\ Uji, Kyoto 611-0011, Japan \\ E-mail: hashida@laser.kuicr.kyoto-u.ac.jp \\ ${ }^{* 2}$ Department of Physics, Graduate School of Science, Kyoto University, Kyoto 606-8502, Japan \\ ${ }^{*}$ FNSPE, Czech Technical University in Prague, 11519 Prague, Czech Republic \\ ${ }^{* 4}$ HiLASE Project, Institute of Physics, ASCR, 18221 Prague, Czech Republic \\ ${ }^{* 5}$ National Institute for Fusion Science, Toki City, Gifu 509-5292, Japan
}

The formation of periodic grating structures has been demonstrated on a titanium surface irradiated by a double-pulse beam with a time delay of $160 \mathrm{fs}$. The first-pulse fluence $F_{\mathrm{PP}}$ was varied and always kept below the threshold $F_{\mathrm{TH}}=60 \mathrm{~mJ} / \mathrm{cm}^{2}$ for forming periodic grating structures on Ti and the delayed pulse fluence $F_{\mathrm{LP}}$ was kept above $F_{\mathrm{TH}}$. The grating structure interspaces were $0.5 \lambda_{\mathrm{L}}$ to $0.85 \lambda_{\mathrm{L}}$ and decreased with $F_{\mathrm{PP}}$ for all values of $F_{\mathrm{LP}}$. This tendency suggests that variation in surface plasma density, which is associated with the fluence of the first pulse, led to variation of the grating interspaces. We found that the interspaces produced by double-pulse irradiation agreed relatively well with those produced by single-pulse irradiation and those predicted by a parametric decay model. To visualize the surface plasma wave induced by the femtosecond laser, two-dimensional particle-in-cell simulation was conducted for a preformed plasma on a metal. The simulation results suggest that the preformed plasma density led to the variation in the grating interspaces. DOI: $10.2961 /$ jlmn.2014.03.0010

Keywords: Periodic grating structures, titanium, femtosecond laser, double-pulse irradiation

\section{Introduction}

On metals under irradiated by linear polarized femtosecond laser pulses, self-organized grating structures are formed [1]-[8] and they are oriented perpendicular to the laser polarization direction. Recently, such grating structures on metal surfaces have been used in several applications, including microfluidics for measuring the protein-folding dynamics [9], surface modification for improving biocompatibility [10], tribology for reducing friction [11][12], surface-enhanced Raman spectroscopy [13], and metal coloring [14][15]. For fluence levels near the ablation threshold, the grating structures have interspaces of $0.5 \lambda_{\mathrm{L}}$ to $0.85 \lambda_{\mathrm{L}}$, which is shorter than the laser wavelength $\lambda_{\mathrm{L}}$. To control the interspaces of the grating structure on metals, the formation mechanism is important. Several models have been proposed for discussing the formation mechanism of these structures. However, the mechanism is not yet understood and remains a topic of debate. The interspaces of the grating structures depended on laser fluence, and this phenomenon is well explained by a parametric decay model [16] proposed by Sakabe et al. To confirm the validity of this model, the interspace dependence on laser fluence has been measured experimentally for $\mathrm{Ti}, \mathrm{Pt}, \mathrm{Mo}, \mathrm{W}, \mathrm{Si}$, and $\mathrm{SiC}$ $[17][18][19]$. We found that the experimental results agreed reasonably well with this model. An assumption in this model is that, as a consequence of the ultrafast interaction with the laser beam, the solid surface is initially covered by a preformed surface plasma with a density much lower than that of the solid. Surface plasma waves are then induced at the interface between free space and the laser-produced low-density plasma by parametric decay of the incident laser light into a surface plasma wave and a scattered electromagnetic wave. The plasma wave travels slowly, at less than $10^{-2}$ times the speed of light, and an ion-enriched local area appears. Before the next electron wave peak arrives, the ions experience a strong Coulomb repulsive force and can be exploded into vacuum; in other words, a Coulomb explosion [20][21][22][23] occurs. Through this process, periodic grating structures are self-organized. Therefore the preformed plasma is a key issue to consider in regard to the formation mechanism of the grating structures. However, it is technically difficult to measure the preformed plasma because its thickness is very small and its density is very low. In this study, a double-pulse irradiation experiment was carried out in order to examine the relation between the preformed plasma density and the grating interspaces self-organized on the metal surface. If the preformed plasma is produced by the rising edge of the laser pulse, an experimental result would be expected in which variation in surface plasma density leads to variation in the grating interspaces. Additionally, we used two-dimensional particle-in-cell simulation to visualize the surface plasma wave.

\section{Experimental results and discussion}

In the experiments, the $T^{6}$-laser system $\left(\lambda_{\mathrm{L}}=805 \mathrm{~nm}, \tau=40\right.$ fs, $10 \mathrm{~Hz}$ ) was used. The experimental setup is shown in Fig. 1. The double-pulse beam with a time delay of $160 \mathrm{fs}$ was composed of a first pulse, responsible for surface plasma formation, and a delayed pulse, responsible for pe- 


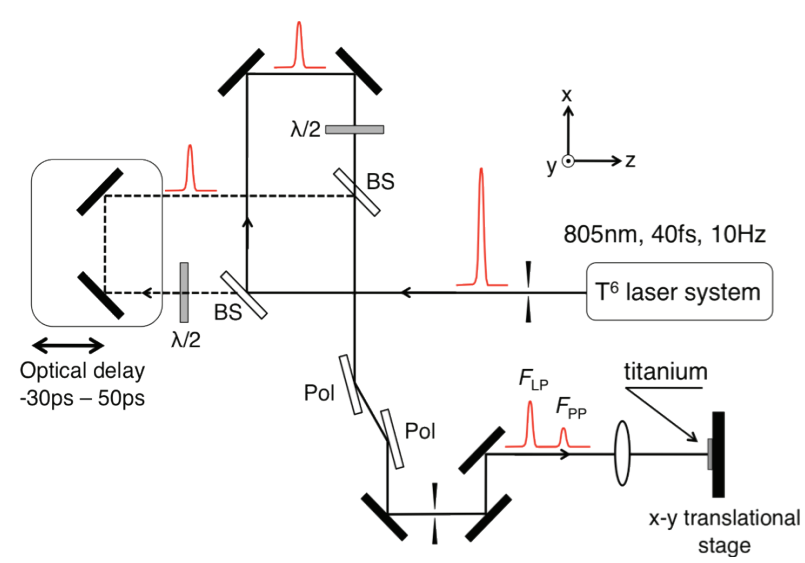

Fig. 1 Experimental setup(BS: beam splitter; Pol: polarizer).

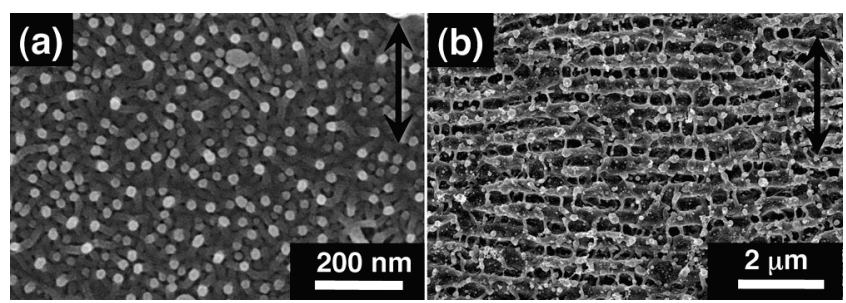

Fig. 2 SEM images of surface structures on titanium produced by (a) 1 pulse and (b) 25 pulses, for double-pulse irradiation with a time delay of $160 \mathrm{fs}$. The laser fluence of the first pulse was $F_{\mathrm{PP}}=$ $45 \mathrm{~mJ} / \mathrm{cm}^{2}$ and that of the delayed pulse was $F_{\mathrm{LP}}=100 \mathrm{~mJ} / \mathrm{cm}^{2}$. Black arrows indicate the laser polarization direction for the double-pulse beam.

riodic grating structure formation. The first-pulse fluence $F_{\mathrm{PP}}$ was varied and always kept below the threshold $F_{\mathrm{TH}}$ $=60 \mathrm{~mJ} / \mathrm{cm}^{2}$ for forming periodic grating structures on Ti. The delayed pulse fluence $F_{\mathrm{LP}}$ was kept constant above $F_{\mathrm{TH}}$. The double pulses were collimated and focused to a spot size of $45 \mu \mathrm{m}$ on the Ti target surface with a lens $(f=10$ $\mathrm{cm})$ at normal incidence in air. The polarization direction of the first pulse was set to be parallel to that of the delayed pulse. The double-pulse beam was shaped to be spatially flat at the target position by image-relaying only the central part of the original Gaussian profile, which was clipped by an aperture and more precisely trimmed by a second aperture [17]. The titanium target was mechanically polished. The surface roughness, Ra, was less than $2 \mathrm{~nm}$ for the sample. The laser fluences in the flat top region were varied in the range $F_{\mathrm{PP}}=10-50 \mathrm{~mJ} / \mathrm{cm}^{2}$ for plasma formation and between the values $F_{\mathrm{LP}}=60,100,150 \mathrm{~mJ} / \mathrm{cm}^{2}$ for periodic grating structure formation. The numbers of double-pulse irradiations was $N=1$ or 25 . Laser-produced grating structures were observed by scanning electron microscopy (SEM; JSM-5560, JEOL).

Figure 2 shows typical surface structures produced by double-pulse irradiations with $N=1$ (a) and $N=25$ (b). For $N$ $=1$, the periodic grating structures did not self-organize but nanostructures approximately $24 \mathrm{~nm}$ in diameter were produced on the irradiated surface. For $N=25$, the periodic grating structures were self-organized in the flat top regions of laser fluence. The grating structures were oriented perpendicular to the laser polarization direction. The nanostructures of approximately $24 \mathrm{~nm}$ in diameter produced when $N=1$ might play a key role in the selforganization of the periodic grating structures when $N=25$. The periodic grating interspaces were determined by read-
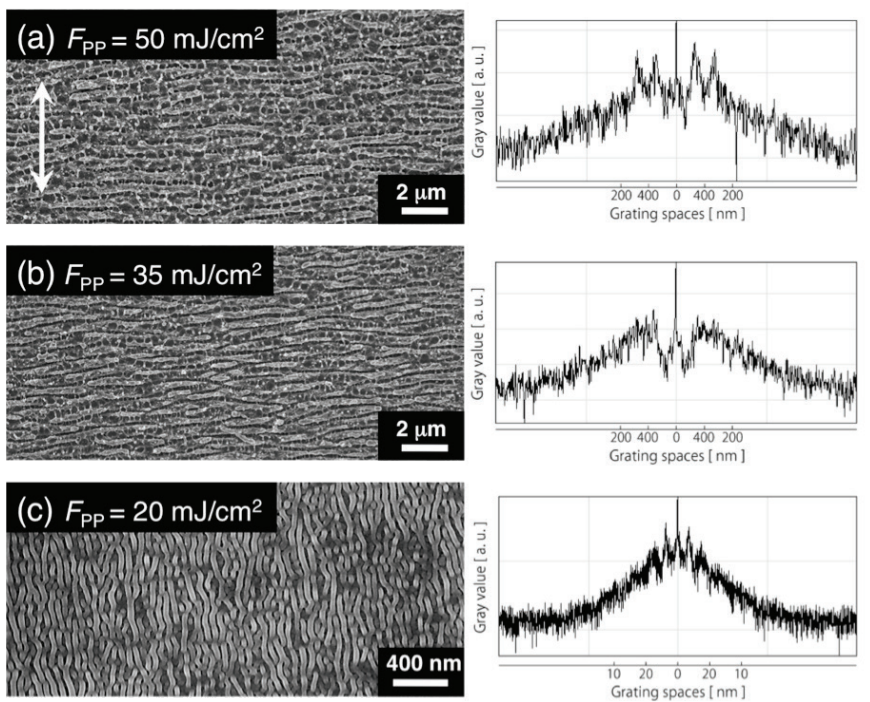

Fig. 3 SEM images of surface structures on titanium produced by 25 pulses of double-pulse irradiation with a time delay of $160 \mathrm{fs}$ at laser fluence of $F_{\mathrm{LP}}=60 \mathrm{~mJ} / \mathrm{cm}^{2}$ (left) and Fourier-transform spectra (right). White arrow shows the laser polarization direction for the double-pulse beam.

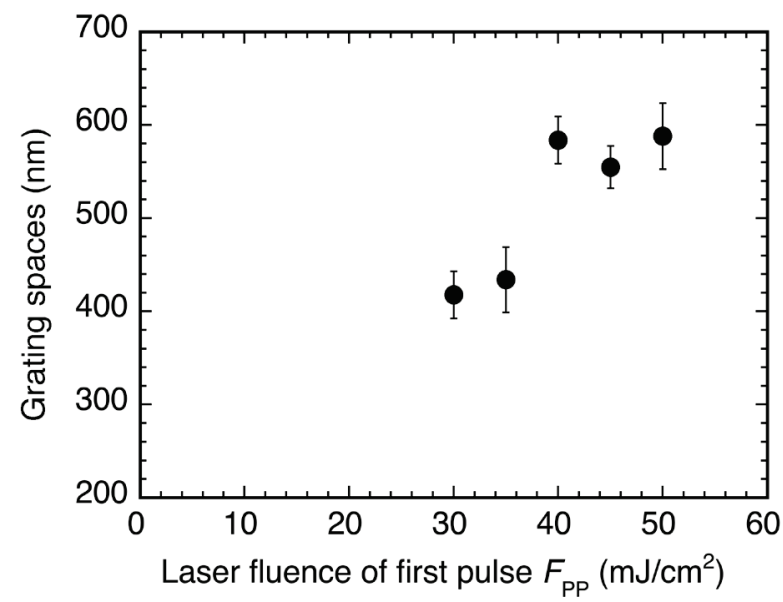

Fig. 4 Dependence of periodic structure interspaces on firstpulse laser fluence $F_{\mathrm{PP}}$ for delayed pulse fluence of $F_{\mathrm{LP}}=60$ $\mathrm{mJ} / \mathrm{cm}^{2}$.

ing the peak value in the frequency domain after Fourier transforming the SEM image of the grating structures. Figure 3 shows SEM images and power spectra of the surface structures produced by varying $F_{\mathrm{PP}}$ while $F_{\mathrm{LP}}$ was kept constant at $60 \mathrm{~mJ} / \mathrm{cm}^{2}$. For $F_{\mathrm{PP}}=50 \mathrm{~mJ} / \mathrm{cm}^{2}$ and $F_{\mathrm{PP}}=35$ $\mathrm{mJ} / \mathrm{cm}^{2}$, the periodic grating structures oriented perpendicular to the laser polarization direction were produced. The interspaces of the periodic grating structures were $604 \mathrm{~nm}$ $\pm 26 \mathrm{~nm}$ for $F_{\mathrm{PP}}=50 \mathrm{~mJ} / \mathrm{cm}^{2}$ and $462 \mathrm{~nm} \pm 28 \mathrm{~nm}$ for $F_{\mathrm{PP}}$ $=35 \mathrm{~mJ} / \mathrm{cm}^{2}$. In contrast, fine ripples [24][25][26] oriented parallel to the laser polarization direction were produced at $F_{\mathrm{PP}}=20 \mathrm{~mJ} / \mathrm{cm}^{2}$. Fine ripples were observed with interspaces of approximately $61 \mathrm{~nm}$, which is much shorter than the range $0.5 \lambda_{\mathrm{L}}$ to $0.85 \lambda_{\mathrm{L}}$ expected under the parametric decay model. Therefore another mechanism appears to contribute to the fine ripple formation. We found that the periodic grating structures were produced when the fluence of the first pulse was higher than $F_{\mathrm{PP}} \geq 35 \mathrm{~mJ} / \mathrm{cm}^{2}$ at a delayed pulse fluence of $60 \mathrm{~mJ} / \mathrm{cm}^{2}$. 


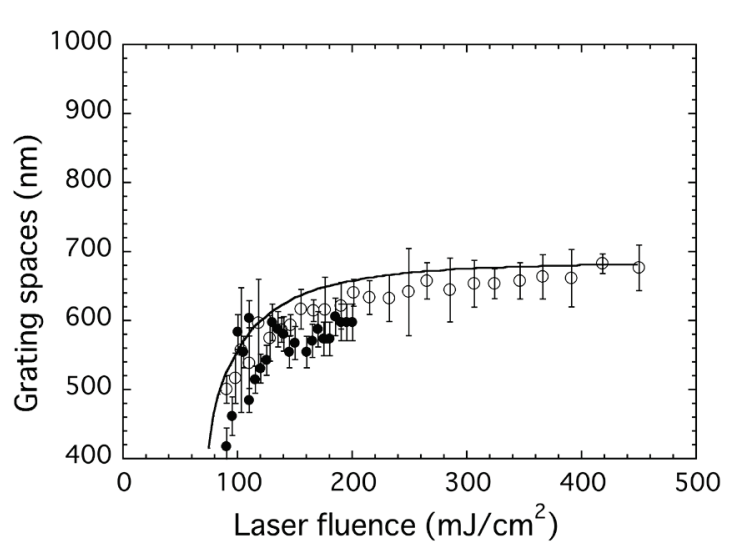

Fig. 5 Laser fluence dependence of periodic structure interspaces produced by femtosecond laser pulses for double pulses with a time delay of $160 \mathrm{fs}$ and for a single pulse with duration of $160 \mathrm{fs}$ [12]. Solid line shows calculation results according to the parametric decay model.

In Fig. 4, the dependence of periodic structure interspaces on first-pulse laser fluence $F_{\mathrm{PP}}$ for the delayed pulse fluence of $F_{\mathrm{LP}}=60 \mathrm{~mJ} / \mathrm{cm}^{2}$. The interspaces of periodic grating structures were in range $0.5 \lambda_{\mathrm{L}}$ to $0.85 \lambda_{\mathrm{L}}$ as expected under the parametric decay model. The interspaces decreased with decreasing $F_{\mathrm{PP}}$. For $F_{\mathrm{LP}}=100 \mathrm{~mJ} / \mathrm{cm}^{2}$ and $F_{\mathrm{LP}}=150$ $\mathrm{mJ} / \mathrm{cm}^{2}$, similar dependence on first-pulse laser fluence was observed. This tendency suggests that variation of the surface plasma density associated with the first-pulse fluence led to a variation of the grating interspaces.

In Fig. 5, the dependence of the periodic structure interspaces on total laser fluence $\left(F_{\mathrm{PP}}+F_{\mathrm{LP}}\right)$ for Ti under double-pulse irradiation is shown (solid circles). For comparison, the periodic grating structures produced by single pulses of $160 \mathrm{fs}$ irradiation [17] are also plotted (open circles). Surprisingly, the interspaces produced by doublepulse irradiation show relatively good agreement with those produced by single-pulse irradiation. Additionally, the interspaces of the periodic structure follow the parametric decay model prediction. The experimental results suggested that the preformed plasma might be produced by the rising edge of the laser pulse, leading to variation in the grating interspaces.

\section{Particle-in-cell simulation of periodic grating struc- ture formation}

Recently, the particle-in-cell simulation results have been reported [27], [28] regarding the dynamics of surface plas- ma wave-induced periodic nanostructuring on solid surfaces. However, the generation mechanism of the surface plasma wave is still under investigation. To visualize the surface plasma wave induced by femtosecond laser pulses, two-dimensional particle-in-cell simulation was performed using the code FISCOF [29][30] for initially preformed plasma on a target. In the simulation, the surface of the preformed plasma was located at $z=2 \mu \mathrm{m}$ (shown by the black dotted line in Fig. 6(a)) and its thickness was $2 \mu \mathrm{m}$ in the $z$ direction of the $(z, y)$ simulation plane. The laser beam was irradiated onto the surface from the left-hand side as indicated by the black arrow. The electron density of the preformed plasma was varied in the range $0-1.0 n_{\mathrm{cr}}$ in $0.1 n_{\text {cr }}$ steps, where $n_{\text {cr }}$ is the critical density for a laser wavelength of $800 \mathrm{~nm}$. The plasma was initially characterized by a Maxwell-Boltzmann distribution with electron temperature $T_{\mathrm{e}}=1 \mathrm{keV}$ and ion temperature $T_{\mathrm{i}}=0.1 T_{\mathrm{e}}$. Hydrogen plasma with $m_{\mathrm{i}} / m_{\mathrm{e}}=1836 / 16$ was used to reduce the calculation time, where $m_{\mathrm{i}}$ and $m_{\mathrm{e}}$ are the ion and electron mass. The charge of the ions was $Z=1$. The formation mechanism of the surface wave is essentially the same for plasma involving a heavy element $Z_{\mathrm{High}}$, though the time for surface wave is longer than that for light elements. However, we have used the electron-hydrogen plasma to reduce the calculation time. The target of $10 n_{\mathrm{cr}}$ was located behind the preformed plasma and it was $10 \mu \mathrm{m}$ thick and $20 \mu \mathrm{m}$ wide. Intense laser light $\left(I=1.0 \times 10^{16} \mathrm{~W} / \mathrm{cm}^{2}, \lambda_{\mathrm{L}}=\right.$ $800 \mathrm{~nm}$, rise time $=15 \mathrm{fs}$ ) was irradiated continuously onto the preformed plasma target at normal incidence. The laser was linearly polarized in the direction parallel to the $y$ axis. Figure 6 shows the electron density distribution at $t=650$ fs for $0.7 n_{\text {cr }}$ of the preformed plasma. The simulation results show that the surface wave is produced on the surface of expanding preformed plasma at $z=1.2 \mu \mathrm{m}$. The surface wave period was analyzed by Fourier transform of the electron density distribution in the area defined by $z=1.0$ to $1.5 \mu \mathrm{m}$ and $y=-5$ to $5 \mu \mathrm{m}$. The obtained FFT spectrum is shown in Fig. 6(b). The surface wave period was about 720 $\mathrm{nm}$ at $0.7 n_{\mathrm{cr}}$ and depended on preformed plasma density $n_{\mathrm{cr}}$. Figure 6(c) shows the interspaces of the surface wave depend on preformed plasma density. Lower preformed plasma density corresponded to shorter interspaces of the surface wave. The obtained simulation results are helpful for discussing the dynamics of surface plasma wave generation. However, the irradiated laser intensity in the simulation is set three orders of magnitude higher than that in the
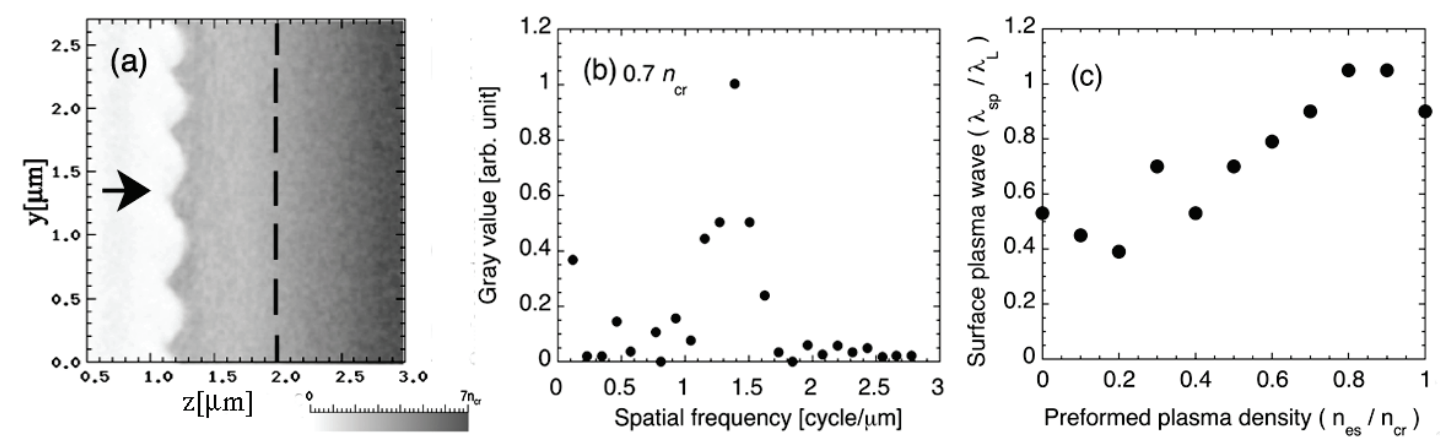

Figure 6 (a) Electron density distribution in the z-y plane at irradiation time of $t=650 \mathrm{fs}$ for $0.7 n_{\mathrm{cr}}$. (b) The Fourier-transform spectrum for the surface electron density of (a) in the area defined by $z=1.0$ to $1.5 \mu \mathrm{m}$ and $y=-5$ to $5 \mu \mathrm{m}$. (c) Dependence of the normalized surface wave on preformed plasma density. 
experiment since the multi-pulse irradiation effect could be taken into account in this 2D-simulation within a practical calculation time. Self-organized periodic grating structures have been observed experimentally as a result of multipulse irradiation in the range of 25-1000 pulses [25][31]. After irradiation of several pulses, the surface of metal is no longer flat. However, nanostructures have been observed distributed as shown in Fig. 1(a) and reported in Ref. [24]. The structures might contribute to enhancing the laser intensity locally through the near-field effect. To reduce this discrepancy, further investigation is needed to capture the accumulation effect for multi-pulse irradiation.

\section{Conclusions}

Grating structure formation has been experimentally demonstrated on a titanium surface irradiated by a doublepulse beam with a time delay of $160 \mathrm{fs}$. We found that the periodic grating structures were produced by first-pulse laser fluence of $F_{\mathrm{PP}} \geq 35 \mathrm{~mJ} / \mathrm{cm}^{2}$ when the delayed pulse fluence was $60 \mathrm{~mJ} / \mathrm{cm}^{2}$. The grating structure interspaces decreased with decreasing $F_{\mathrm{PP}}$ for all $F_{\mathrm{LP}}$ values used. This tendency suggests that variation of the surface plasma density associated with the first-pulse fluence led to variation in the grating interspaces. The interspaces produced by double-pulse irradiation showed relatively good agreement with those produced by single-pulse irradiation with pulse duration of $160 \mathrm{fs}$ and those predicted by the parametric decay model. To visualize the surface plasma wave induced by the femtosecond laser, two-dimensional particlein-cell simulation was performed for initially preformed plasma on a metal. The simulation results suggest that the preformed plasma density led to variation in the grating interspaces.

\section{Acknowledgments}

We thank T. Kanaya, K. Nishida, and T. Terashima for their assistance with SEM and laser scanning microscopy. This study was financially supported by a Grant-in-Aid for Scientific Research (C)(25420728) from the Ministry of Education, Culture, Sports, Science and Technology (MEXT), Japan, the Amada Foundation for Metal Work Technology, and NIFS Collaborative Research Program (NIFS11KNTS013) and was partially supported by both a Grant-in-Aid for the Global COE program (Next Generation of Physics, Spun from Universality and Emergence from MEXT, Japan) and the ICR-KU International ShortTerm Exchange Program for Young Researchers. This work benefitted from the support of the Czech Science Foundation (Project P205/11/1165), the Czech Republic's Ministry of Education, Youth, and Sports through the HiLASE Project (CZ.1.05/2.1.00/01.0027) and DPSSLasers Project (CZ.1.07/2.3.00/20.0143) co-financed from the European Regional Development Fund. This research was partially supported by the grant RVO 68407700 .

\section{References}

[1] M. Hashida, M. Fujita, M. Tsukamoto, A. Semerok, et al.: Proc. SPIE 4830, pp.452-457(2003).
[2] M. Tsukamoto, K. Asuka, H. Nakano, M. Hashida et al.: Vacuum 80, 1346-1350(2006).

[3] A. Y. Vorobyev, V. S. Makin, and C. Guo: J. Appl. Phys. 101 (2007)034903.

[4] J. Wang and C. Guo: Appl. Phys. Lett. 87, 251914 (2005).

[5] A. Y. Vorobyev and C. Guo: Phys. Rev. B 72, 195422 (2005).

[6] A. Weck, T. H. R. Crawford, D. S. Wilkinson, et al.: Appl. Phys. A 90, 537-543 (2008).

[7] B. K. Nayak, M. C. Gupta, and K. W. Kolasinski: Appl. Phys. A 90, 399-402 (2008).

[8] Y. Huang, S. Liu, W. Li, et al.: Opt. Express 17, 20756-20761 (2009)

[9] S. Matsumoto, A. Yane, S. Nakashima et al.: J. Am. Chem. Soc. 129, 3840-3841(2007).

[10] T. Shinonaga, M. Tsukamoto, A, Nagai, K. Yamashita, T, Hanawa, N. Matsushita, G. Xie, N. Abe: Appl. Surf. Sci. 288, 649-653 (2014).

[11] T. Kato and N. Abe: Rev. Laser Eng. 37, 510(2009).

[12] N. Yasumaru, K. Miyazaki, J. Kiuchi: Appl. Surf. Sci. 254, 2364(2008).

[13] C. Wang, Y. Chang J. Yao, C. Luo, S. Yin, et al.: Appl. Phys. Lett. 100, 023107(2012).

[14] A. Vorobyev and C. Guo: Laser Photonics Rev. 7, 385 (2013).

[15] M. Kawamoto, M. Hashida, Y. Miyasaka, M. Shimizu, et al. : IEEJpn., LAV-13-016, pp.7-10 (2013).

[16] S. Sakabe, M. Hashida, S. Tokita, S. Namba, et al,: Phys. Rev. B 79, 033409(2009).

[17] K. Okamuro, M. Hashida, Y. Miyasaka, Y. Ikuta, et al,: Phys. Rev. B 82, 165417(2010).

[18] M. Hashida, Y. Ikuta, Y. Miyasaka, S. Tokita, et al.: Appl. Phys. Lett.102, 174106(2013).

[19] L. Gemini, M. Hashida, M. Shimizu, Y. Miyasaka, et al.: J. Appl. Phys.114, 194903(2013).

[20] S. Sakabe, K. Shirai, M. Hashida, S. Shimizu, et al.: Phys. Rev. A 74, 043205(2006).

[21] M. Hashida, S. Namba, K. Okamuro, S. Tokita, et al.: Phys. Rev. B 81 (2010)115442.

[22] Y. Miyasaka, M. Hashida, Y. Ikuta, K. Otani, et al.: Phys. Rev. B 86, 075431(2012).

[23] M. Hashida, Y. Miyasaka, Y. Ikuta, S. Tokita, et al.: Phys. Rev. B 83 (2011)235413.

[24] M. Shimizu, M. Hashida, Y. Miyasaka, S. Tokita, and S. Sakabe: Appl. Phys. Lett.103, 174106(2013).

[25] J. Bonse, J. Krüger, S. Höhm, and A. Rosenfeld: J. Laser Appl. 24, 042006 (2012).

[26]E. V. Golosov, V. I. Emel'yanov, A.A. Ionin, Y. R. Kolobov, et al.: JETP Lett. 90, 107-110 (2009).

[27] M. Djouder, T. E. Itina, D. Deghiche, et al.: Appl. Surf. Sci. 258, 2580-2583 (2012).

[28] M. Djouder, O. Lamrous, M. D. Mitiche, et al.: Appl. Surf. Sci. 258, 711-714 (2013).

[29] H. Sakagami and K. Mima: Proc. 2nd Int. Conf. Inertial Fusion Sci. Appl. (2001)380-383.

[30] T. Ogata, et al.: Meeting Abstracts Phys. Soc. Jpn. 67, Issue 1, Part 4(2012)pp.947.

[31] M. Hashida, Y. Miyasaka, Y. Ikuta, K. Otani, S. Tokita, and S. Sakabe: J. Laser Micro/Nanoengineering 7(2012)194-197.

(Received: April 15, 2014, Accepted: September 05, 2014) 